\title{
ERK1/2 mediates glucose-regulated POMC gene expression in hypothalamic neurons
}

\author{
Juan Zhang, Yunting Zhou, Cheng Chen, Feiyuan Yu' ${ }^{1}$, Yun Wang', Jiang Gu', \\ Lian Ma and Guyu Ho \\ Department of Pediatrics, The Second Affiliated Hospital of Shantou University Medical College, \\ Shantou 515041, China \\ 1Department of Molecular Pathology, Shantou University Medical College, Shantou 515041, China
}

Correspondence should be addressed to $\mathrm{G} \mathrm{Ho}$

Email

guyuh@yahoo.com

\begin{abstract}
Hypothalamic glucose-sensing neurons regulate the expression of genes encoding feeding-related neuropetides POMC, AgRP, and NPY - the key components governing metabolic homeostasis. AMP-activated protein kinase (AMPK) is postulated to be the molecular mediator relaying glucose signals to regulate the expression of these neuropeptides. Whether other signaling mediator(s) plays a role is not clear. In this study, we investigated the role of ERK1/2 using primary hypothalamic neurons as the model system. The primary neurons were differentiated from hypothalamic progenitor cells. The differentiated neurons possessed the characteristic neuronal cell morphology and expressed neuronal post-mitotic markers as well as leptin-regulated orexigenic POMC and anorexigenic AgRPINPY genes. Treatment of cells with glucose dose-dependently increased $P O M C$ and decreased AgRPINPY expression with a concurrent suppression of AMPK phosphorylation. In addition, glucose treatment dose-dependently increased the ERK1/2 phosphorylation. Blockade of ERK1/2 activity with its specific inhibitor PD98059 partially (approximately 50\%) abolished glucose-induced POMC expression, but had little effect on AgRPINPY expression. Conversely, blockade of AMPK activity with its specific inhibitor produced a partial (approximately 50\%) reversion of low-glucose-suppressed POMC expression, but almost completely blunted the low-glucose-induced AgRP/NPY expression. The results indicate that ERK1/2 mediated POMC but not AgRP/NPY expression. Confirming the in vitro findings, i.c.v. administration of PD98059 in rats similarly attenuated glucoseinduced POMC expression in the hypothalamus, but again had little effect on AgRPINPY expression. The results are indicative of a novel role of ERK $1 / 2$ in glucose-regulated POMC expression and offer new mechanistic insights into hypothalamic glucose sensing.
\end{abstract}

Journal of Molecular Endocrinology (2015) 54, 125-135

\section{Introduction}

The body's nutritional state is sensed by the brain, whereby feed-back signals are generated to maintain metabolic homeostasis (Blouet \& Schwartz 2010, Jordan et al. 2010). The hypothalamus, particularly the arcuate nucleus (ARC) where feeding-related neuropeptides
Key Words
- ERK $1 / 2$
- AMPK
- POMC
- AgRP
- NPY
- glucose sensing 
Results from previous studies indicate that infusion of glucose into the hypothalamus leads to decreased food intake (Kurata et al. 1986), suggesting that one function of hypothalamic glucose sensing is appetite control. Consistent with this observation, systemic or i.c.v. administration of glucose stimulates POMC and inhibits AgRP/NPY expression (Chang et al. 2005, Wolfgang et al. 2007, Cha et al. 2008). Glucose-regulated AgRP or POMC expression has also been demonstrated in transformed cell lines (Lee et al. 2005, Cai et al. 2007, Cheng et al. 2008). Because the glucose-regulated gene expression can be blunted by the non-metabolizable glucose analogue 2-deoxyglucose in vivo as well as in cell lines (Lee et al. 2005, Wolfgang et al. 2007, Cha et al. 2008, Cheng et al. 2008), the results indicate that intracellular glucose metabolism is required for the expression of these genes.

AMP-activated protein kinase (AMPK) is a cellular energy sensor, activated under states of low cellular energy such as glucose deprivation (Kahn et al. 2005, Hardie et al. 2006). AMPK is also postulated to mediate hypothalamic glucose sensing. For instance, i.c.v. injection of glucose suppresses hypothalamic AMPK activity (Minokoshi et al. 2004, Cha et al. 2008); virally mediated expresison of activated or dominant-negative mutants of AMPK in the hypothalamus modulates AgPR/NPY expression in the fasted or fed state (Minokoshi et al. 2004); expression of dominant-negative mutants of AMPK in cell lines also attenuates low-glucoseinduced AgRP expression (Lee et al. 2005). In agreement with the regulatory role of AMPK in orexigenic neuroupeptide expression, transgenic mice with AgRP-neuron-specific AMPK inactivation display decreased feeding and a lean phenotype (Claret et al. 2007). However, evidence supporting the role of AMPK in POMC expression is perplexing. For instance, mice with POMC-neuron-specific deletion of AMPK (POMC $\alpha 2 \mathrm{KO}$ ), which would be predicted to have enhanced Pomc expression and thus a lean phenotype, are hyperphagic and obese (Claret et al. 2007); virally mediated expression of dominant-negative mutants of AMPK in the hypothalamus also produces a paradoxical suppression of POMC expression in the fed state (Minokoshi et al. 2004). Furthermore, using pharmacological inhibitors it has been demonstrated that AMPK mediates the glucose-inhibited electrical activity of hypothalamic NPY-expressing neurons, but its role in the activity of glucose-excited neurons (e.g., POMC neurons) is unclear (Mountjoy et al. 2007). However, POMC neurons from Pomc 2 2KO mice show defective electrophysiology (Claret et al. 2007). It appears that the molecular mechanism governing glucose sensing in POMCexpressing neurons is complex and that other signaling mediator(s) might be involved.
Pancreatic islet cells are prototypic glucose-sensing cells (Schuit et al. 2001, Mountjoy \& Rutter 2007). In $\beta$-cells, glucose not only induces insulin secretion but also insulin expression (Lawrence et al. 2008), the latter event is mediated by ERK1/2 (Khoo et al. 2003). Hypothalamic ERK1/2 has been implicated in energy homeostasis. Fasting activates ERK1/2 in the ARC and paraventricular nucleus in mice (Morikawa et al. 2004, Ueyama et al. 2004) and the activation is reversed by refeeding (Ueyama et al. 2004). Hypothalamic ERK $1 / 2$ has also been shown to mediate the anorectic and thermogenic effect of leptin (Rahmouni et al. 2009) and the leptin-induced glucose utilization (Toda et al. 2013).

To address whether ERK1/2 plays a role in hypothalamic glucose sensing, we generated primary hypothalamic neurons via differentiation of rat hypothalamic progenitor cells. The cultured cells exhibited neuronal cell morphology and expressed the neuron-specific cellular marker $\beta$ III tubulin ( $\beta I I I)$ and post-mitotic marker NeuN, confirming the neuronal nature and maturation state. Using these primary neurons, we investigated the contribution of ERK1/2 to glucose-regulated POMC and AgRP/NPY expression.

\section{Materials and methods}

\section{Animals}

Sprague-Dawley (SD) rats were purchased from the Animal Center of Shantou University Medical College. The animals were bred and housed under standard conditions with food and water available and the animals allowed to eat and drink ad libitum. All experimental procedures were conducted in accordance with the guidelines published in the Ministry of Science and Technology of China for Care and Use of Laboratory Animals and approved by the Animal Care and Welfare Committee of Shantou University Medical College.

\section{Hypothalamic cell isolation and primary cell culture}

Timed-pregnant SD rats on day 18 of gestation (E18) were killed under anesthesia. Fetal brains were collected and hypothalami dissected as described previously (Bergonzelli et al. 2001). Hypothalamic cells were isolated according to a published method (Sousa-Ferreira et al. 2011) with modifications. Fetal hypothalami were immediately placed into Hank's Balanced Salt Solution (HBSS; Macgene, Beijing, China), washed with HBSS, cut into 5-10 fragments, and digested in Neurobasal medium

Published by Bioscientifica Ltd. 
(Gibco) containing $2 \mathrm{mg} / \mathrm{ml}$ papain (Sigma) and $20 \mathrm{U} / \mathrm{ml}$ DNAase I (Biotechnology Center, Guwahati, India) for $30 \mathrm{~min}$ at $37^{\circ} \mathrm{C}$. The tissue fragments were then mechanically dissociated with a sterile Pasteur pipette by gently pipetting them up and down for ten times. After sitting for $2 \mathrm{~min}$, the supernatant was aspirated and residual tissue fragments were washed with Neurobasal medium twice by pipetting up and down as before. The pooled supernatants were centrifuged at $500 \boldsymbol{g}$ for $2 \mathrm{~min}$ and the cell pellets resuspended in Neurobasal medium. Viable hypothalamic cells were counted using Trypan Blue staining and plated into poly-D-lysine (Sigma)-coated six-well culture plates at density of $2 \times 10^{6}$ cells per well or $2 \times 10^{5}$ cells per well containing coverslips. The cells were incubated at $37^{\circ} \mathrm{C}$ with $5 \% \mathrm{CO}_{2}$ in the differentiation medium containing Neurobasal, $500 \mathrm{mM}$ L-glutamine, 2\% B27 supplement, and 2\% penicillin-streptomycin (all from Gibco) to allow for neuronal differentiation. Neurobasal medium contained $25 \mathrm{mM}$ glucose throughout the study unless specified otherwise.

\section{Immunocytochemistry}

DIV10 cells were washed with PBS, fixed in 4\% paraformaldehyde, and permeabilized with $0.3 \%$ Triton X-100 (Sigma). The cells were then blocked with 3\% BSA (Sigma) for $1 \mathrm{~h}$, followed by incubation overnight at $4{ }^{\circ} \mathrm{C}$ using a blocking buffer containing each of the following primary antibodies: rabbit anti-NeuN (1:5000, Cell Signaling Technology, Beverley, MA, USA), and mouse anti-BIII tubulin (1:200, Millipore). Following washes with PBS, the cells were incubated for $1 \mathrm{~h}$ at room temperature with secondary antibodies of goat anti-mouse Alexa-Flour 594 or goat anti-rabbit Alexa-Flour 488 (1:200, ZS-Bio, China). The cells were then stained with the nuclear dye DAPI (Vectashield, Burlingame, CA, USA) for $5 \mathrm{~min}$. Coverslips were mounted on microscope slides and fluorescence images captured using a fluorescence microscope (LSM510 Meta (Zeiss, Jena, Germany)). The percentage of antibodypositive cells was calculated using the number of doublepositive cells divided by the number of DAPI-positive cells in four independent microscopic fields (approximately 100 cells) per coverslip. At least three independent cell culture preparations were analyzed for each treatment.

\section{Pharmacological treatment in vitro}

E18 progenitor cells were cultured in the differentiation medium for 10 days (DIV10) and subjected to pharmacological treatments as specified in the Results section.
Rat leptin was obtained from Biovendor (Brno, Czech Republic). Glucose, compound C (CC), and PD098059 were purchased from Sigma. CC and PD098059 were dissolved in DMSO as $10 \mathrm{mM}$ stocks, such that the final DMSO concentration used in cell culture was $0.2 \%$.

\section{Intracerbroventricular (i.c.v.) injection}

SD rats (8-10 weeks of age) provided with standard rodent chow and allowed to feed at libitum were food-deprived overnight and randomized into treatment and control group ( $n=4 /$ group). The rats were then anesthetized and sterotaxically given i.c.v. injections of $10 \mu \mathrm{mol}$ glucose in $2 \mu \mathrm{l}$ saline. PD98059 ( 2.5 or $5 \mu \mathrm{g}$ in $2 \mu \mathrm{l}$ DMSO) was given i.c.v. $30 \mathrm{~min}$ before glucose administration. The control group was given equivalent volumes of the vehicles. After $1 \mathrm{~h}$ of glucose administration, rats were killed and ARC regions of hypothalami were collected for RNA and protein extraction.

\section{Quantitative PCR}

Hypothalami from E18, and postnatal days 0 and 28 (P0 and P28) were homogenized on ice in HBSS and cultured hypothalamic cells were washed with PBS on ice before RNA extraction. Total cellular RNAs were extracted using TRIzol (Life Technologies) according to the manufacturer's instructions and RNA purity was confirmed by the $260 / 280$ absorption ratio ( $>1.8$ ). cDNAs were prepared from $1 \mu \mathrm{g}$ total RNAs using PrimeScript RT with gDNA eraser (Takara, Tokyo, Japan) according to the manufacturer's instructions. Quantitative PCRs (qPCRs) were performed using SYBR Premix Ex TaqII (Takara). The primers used were as follows $\left(5^{\prime}-3^{\prime}\right)$ : $\beta$-actin (forward: ACCCTGAAGTACCCCATTG; reverse: TACGACCAGAGGCATACAG), Npy (forward: GACATGGCCAGATACTACTCCG; reverse: AAGGGTCTTCAAGCCTTGTTCT), Agrp (forward: GCAGAGGTGCTAGATCCACAGAA; reverse: AGGACTCGTGCAGCCTTACAC; Chang et al. 2004), Pomc (forward: AGTGCCAGGACCTCACCACG; reverse: AAGCGGTCCCAGCGGAAG; Martins et al. 2010), LepR (forward: ACAGCCAAACAAAAGCACCATT; reverse: TCAGCAGCCTCTCTTACGATTG). Reactions were run in the 7500 Fast Real-Time PCR System (Applied Biosystems) under the following conditions: $95^{\circ} \mathrm{C}$ for $15 \mathrm{~min}$, followed by 40 cycles of $94{ }^{\circ} \mathrm{C}$ for $15 \mathrm{~s}, 55^{\circ} \mathrm{C}$ for $30 \mathrm{~s}$, and $72^{\circ} \mathrm{C}$ for $30 \mathrm{~s}$. Each sample was run in triplicate. Quantification of relative mRNA levels was performed using the change in threshold cycles $(\Delta C \mathrm{t})$ method with $\beta$-actin as the internal reference with the 7500 Software v2.0.6 (Applied Biosystems).

Published by Bioscientifica Ltd. 


\section{Western blotting analysis}

Proteins were extracted from cultured cells using RIPA buffer (CellSignaling Technology) containing $1 \mathrm{mMNaF}$ and $1 \mathrm{mM}$ PMSF (Solarbio, Beijing, China). Protein concentrations were determined using the BCA assay (Thermo Scientific, Waltham, MA, USA). Equivalent amounts of protein samples were separated by 10\% SDS-PAGE and transferred to nitrocellulose membranes (Whatman, Dusseldorf, Germany). The membranes were incubated with 5\% BSA in TBST at room temperature to block nonspecific binding and then incubated with primary antibodies of rabbit antiphospho-AMPK $\alpha$ (Thr172) or rabbit anti-phospho-ERK1/2 (Thr202/Tyr204) (1:1000, Cell Signaling Technology), or mouse anti- $\beta$-actin (1:2000, ZS-Bio) overnight at $4{ }^{\circ} \mathrm{C}$. Subsequently, the membranes were incubated with secondary antibodies (IRDye 680RD-conjugated goat anti-rabbit or IRDye $800 \mathrm{CW}$-conjugated goat anti-mouse (1:10 000, LI-COR, USA)) for $1 \mathrm{~h}$ at room temperature. The band density was visualized, scanned, and analyzed using the Odyssey Two-Color Infrared Imaging System (LI-COR) and quantified using the Quantity OneSystem (Bio-Rad).

\section{Statistical analysis}

Student's $t$-test was used throughout the study. Data were expressed as the mean \pm s.e.M. Differences with $P<0.05$ were considered to be statistically significant.

\section{Results}

\section{Characterization of cultured hypothalamic neurons}

Primary hypothalamic neurons were generated by differentiating rat hypothalamic progenitor cells collected on embryonic day 18 (E18). Upon ex vivo differentiation, we saw a time-dependent increase in the expression of Agrp, Npy, Pomc, and leptin receptor (LepR) genes, peaking at around 10 days (DIV10) in culture (Fig. 1A) and the expression levels
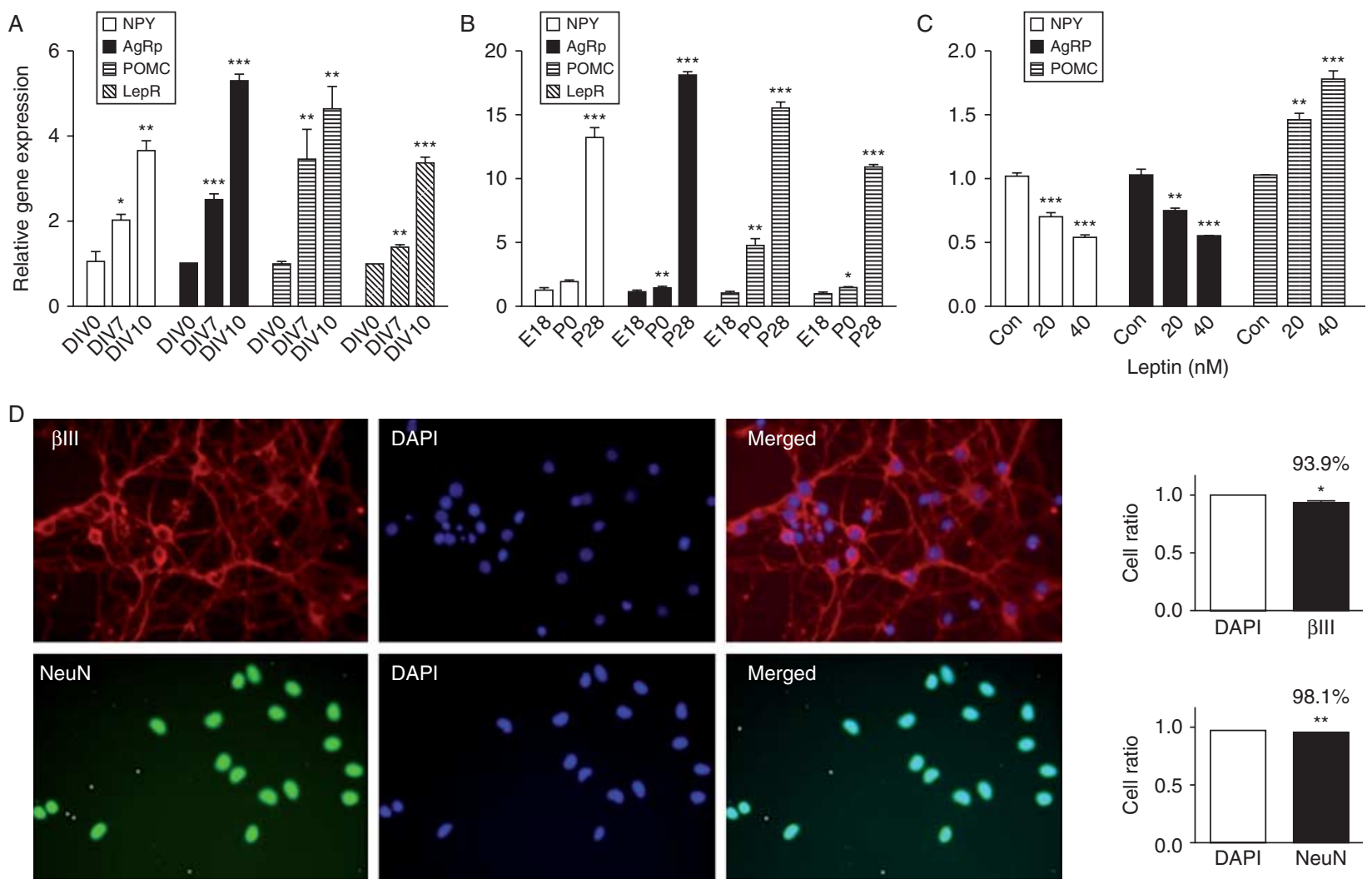

\section{Figure 1}

Characteristics of differentiated hypothalamic neurons. (A) Hypothalamic progenitor cells were grown in differentiation medium for 0,7 , and 10 days and mRNAs were isolated for the analyses of expression of the indicated genes expression by qPCR. (B) Hypothalamic mRNAs isolated from E18, P0, and P28 rats were analyzed identically to those examined in A. (C) DIV10 neurons were cultured in Neurobasal medium containing $5 \mathrm{mM}$ glucose
(C) 2015 Society for Endocrinology Printed in Great Britain overnight before being treated with 20 or $40 \mathrm{nM}$ leptin for $1 \mathrm{~h}$. Cellular mRNAs were isolated for gene expression analysis. (D) DIV10 neurons were stained with antibodies against $\beta I I I$ and NeuN and double-stained with the nuclear dye DAPI. All experiments were independently carried out at least three times and the results are representative. ${ }^{\star} P<0.05$, ${ }^{*} P<0.01$, and $* * * P<0.001$ 
declined thereafter (results not shown). DIV10 cells were therefore chosen for further characterization. A 3.7- to 5.3fold increase in the expression of the genes was found on DIV10 compared with the levels on day 0 (DIV0) in culture (Fig. 1A). As for comparison, rat hypothalamic mRNAs from developmental stages of E18, postnatal day 0 (P0), and 28 (P28) were analyzed. A 1.3- to 4.5- and 11- to 18-fold increase was found at P0 and P28, respectively, compared with those at E18 (Fig. 1B). As DIV0 cells (freshly isolated from E18) and E18 hypothalami were similar with respect to gene expression (results not shown), DIV10 cells appeared to have expression levels approximating to those of neonatal rats.

Leptin regulates $P O M C$ and $A g R P / N P Y$ gene expression in an opposing manner (Barsh \& Schwartz 2002, Varela \& Horvath 2012). Consistently, DIV10 cells exhibited a dosedependent decrease in Agrp/Npy and increase in Pomc expression upon leptin challenge (Fig. 1C), indicating that DIV10 cells are leptin-sensitive.

The neuronal homogeneity and maturity of DIV10 cells were examined using the neuron-specific cellular marker $\beta I I I$ and post-mitotic marker NeuN. $\beta$ III-positive cells exhibited an exclusive cytoplasmic staining with long cellular processes characteristic of neurons: $94 \%$ of cells were double-positive for $\beta I I I$ and DAPI (Fig. 1D). NeuN-positive cells displayed an exclusive nuclear staining and $98 \%$ of cells were double-positive for NeuN and DAPI (Fig. 1D).

\section{Glucose modulates the expression of feeding-related neuropeptides in DIV10 neurons}

To assess the effect of glucose on the expression of feedingrelated neuropeptides, DIV10 neurons were treated with increasing glucose concentrations of 1, 5, 10, $25 \mathrm{mM}$ for $1 \mathrm{~h}$. The Pomc mRNA levels were dose-dependently increased, with a 2.4-fold elevation with $25 \mathrm{mM}$ glucose compared with the $1 \mathrm{mM}$ glucose treatment (Fig. 2). Conversely, the Agrp and Npy expression was dose-dependently decreased, with a respective $65 \%$ and $60 \%$ reduction for treatment with $25 \mathrm{mM}$ glucose compared with the $1 \mathrm{mM}$ treatment (Fig. 2). The regulatory effect of glucose on the orexigenic and anorexigenic neuropeptide expression in DIV10 neurons agrees with the results reported previously (Chang et al. 2005, Lee et al. 2005, Cai et al. 2007, Wolfgang et al. 2007, Cha et al. 2008, Cheng et al. 2008).

\section{Glucose activates ERK1/2 in DIV10 neurons}

To investigate the role of ERK1/2 in glucose sensing, DIV10 neurons were treated with increasing concentrations of glucose for $1 \mathrm{~h}$ and the ERK1/2 phosphorylation
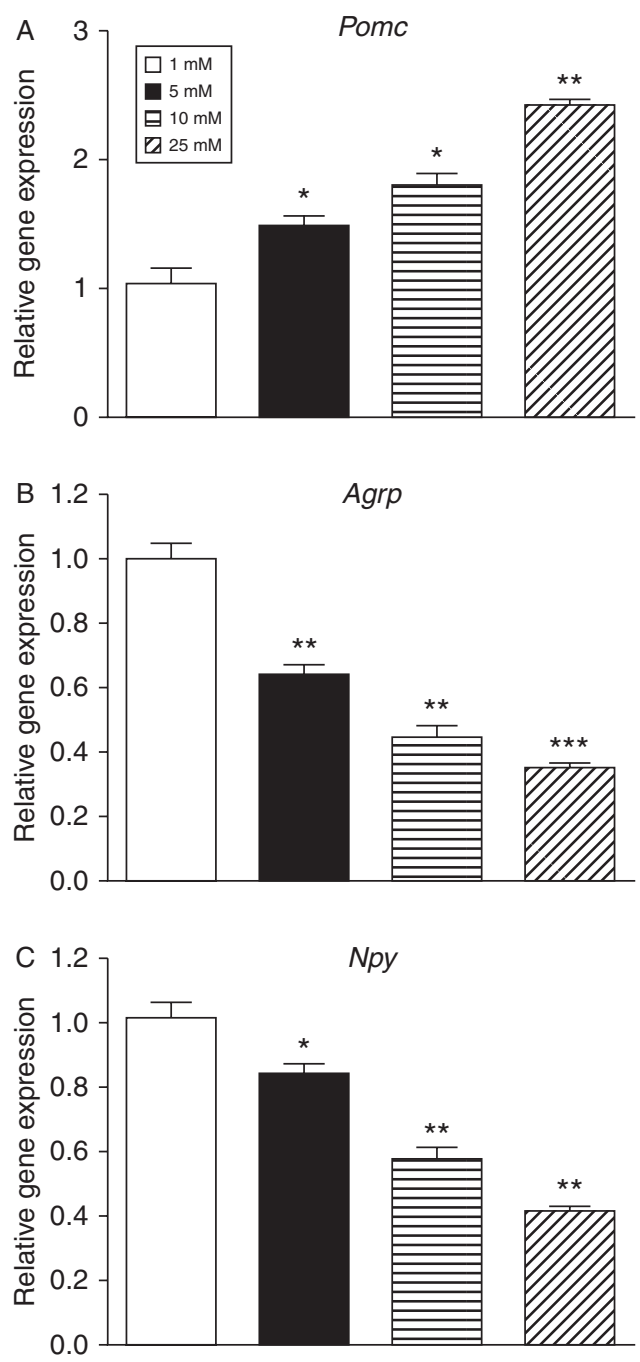

Figure 2

Glucose regulates the Pomc and Agrp/Npy gene expression in DIV10 neurons. DIV10 neurons were incubated in Neurobasal medium containing $1 \mathrm{mM}$ glucose overnight before being treated with the indicated concentrations of glucose for $1 \mathrm{~h}$. Cellular mRNAs isolated from each treatment were subjected to qPCR analyses. $* P<0.05, * * P<0.01$, and $* * * P<0.001$ compared with cells treated with $1 \mathrm{mM}$ glucose.

was examined. A dose-dependent increase in phosphorylated ERK1/2 (pERK1/2) was seen, which was paralleled by a dose-dependent reduction in phosphorylated AMPK (pAMPK) (Fig. 3). The results indicate that glucose, in addition to inhibiting AMPK as expected, activates ERK1/2 in DIV10 neurons.

\section{Contribution of ERK1/2 to Pomc expression in glucose sensing}

To establish that ERK1/2 mediates the appetitive gene expression in glucose sensing, the MEK (upstream

Published by Bioscientifica Ltd 

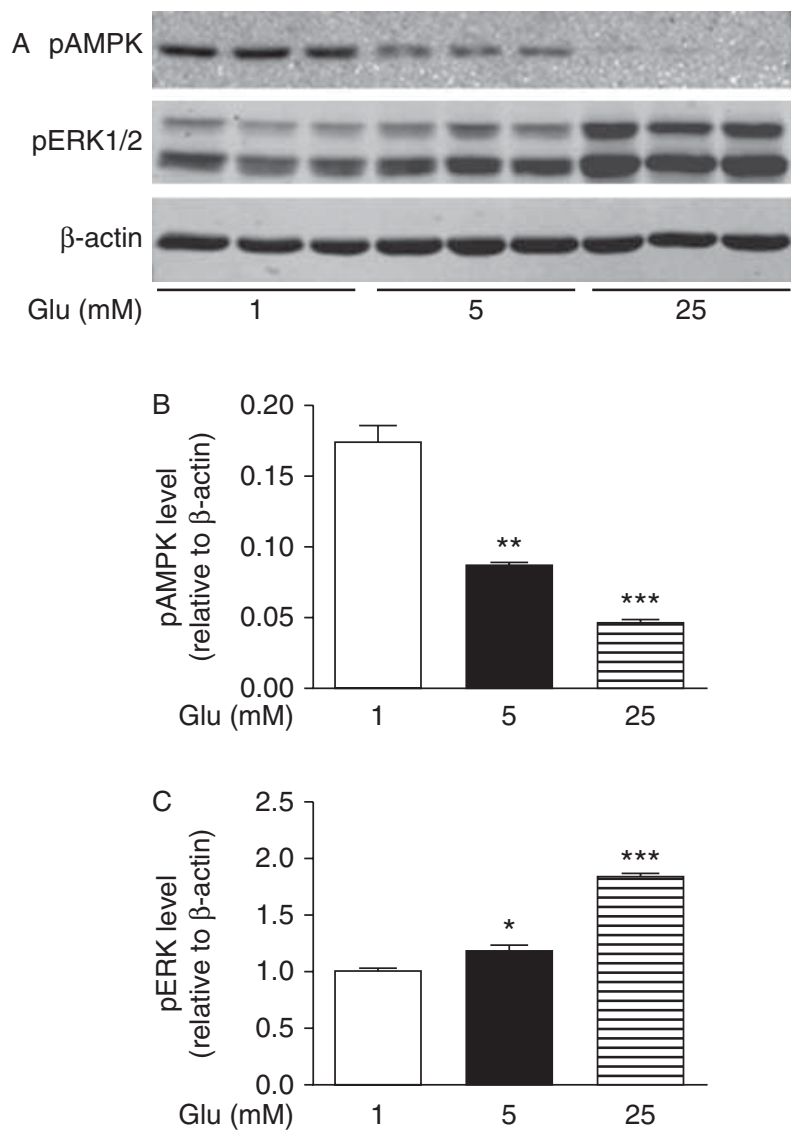

Figure 3

Glucose inhibits AMPK and promotes ERK1/2 phosphorylation in DIV10 neurons. DIV10 neurons were incubated in Neurobasal medium containing $1 \mathrm{mM}$ glucose overnight, followed by 1,5 , and $25 \mathrm{mM}$ glucose (Glu) treatment for $1 \mathrm{~h}$. The PAMPK and pERK $1 / 2$ levels were analyzed by western blotting and quantified by densitometry scan. $* P<0.05, * * P<0.01$ and $* * * P<0.001$ compared with cells treated with $1 \mathrm{mM}$ glucose.

kinase of ERK1/2)-specific inhibitor PD98059 was used to modulate ERK1/2 activity. The inhibitor dose-dependently blocked glucose-induced pERK1/2 levels (Fig. 4A). PD98059 at $10 \mu \mathrm{M}$ suppressed the pERK1/2 level by $71 \%$ (Fig. 4A) while it produced a $28 \%$ reduction in glucosestimulated Pomc expression (Fig. 4B). PD98059 at $20 \mu \mathrm{M}$, which depressed glucose-induced pERK1/2 levels by $125 \%$, further blunted the Pomc expression by 53\% (Fig. 4B). In contrast, PD98059 at $10 \mu \mathrm{M}$ had no effect on glucose-suppressed Agrp/Npy expression ( $P>0.05$, Fig. 4B). At $20 \mu \mathrm{M}$, at which the pERK1/2 level was inhibited below basal level (125\%), PD98059 only produced a modest attenuation of $15-17 \%$ (Fig. 4B). Because PD98059 is a highly specific ERK1/2 inhibitor with few known off-target effects even when used at $50 \mu \mathrm{M}$ (Alessi et al. 1995), the results indicate that ERK1/2 partially participates in glucose-regulated Pomc expression, but its role in Agrp/Npy expression, if any, is minimal. The results also indicate the existence of additional regulatory pathway(s) for Pomc expression.

\section{Contribution of AMPK to Pomc expression in glucose sensing}

Although glucose coregulates Pomc expression and AMPK activity in vivo (Minokoshi et al. 2004, Cha et al. 2008), no clear evidence yet indicates AMPK to be the mediator relaying glucose signals to Pomc expression. To address this question, the AMPK-specific inhibitor CC was employed to modulate its activity. CC dose-dependently inhibited low-glucose-induced elevation of AMPK phosphorylation, achieving a $100 \%$ inhibition $(P>0.05)$ at $20 \mu \mathrm{M}$. At this inhibitor concentration, the low-glucosesuppressed Pomc expression was attenuated by 55\% (Fig. 5B). In contrast, the low-glucose-induced Agrp/Npy expression was almost completely (approximately 90\%) abolished (Fig. 5B). Although CC may have off-target effects, the effects influencing the results observed here might be minimal, because i) similar CC concentrations have been used in cultured hypothalamic neurons, which showed no apparent off-target effects (Mountjoy et al. 2007) and ii) CC modulated the Pomc and Agrp/Npy expression in manners (i.e., opposite directions) consistent with its specific effect. Thus, the results indicate that AMPK is the primary mediator of glucose-regulated Agrp/Npy expression, but only a partial contributor to Pomc expression, which supports the hypothesis that ERK is an additional mediator relaying glucose signals to anorexigenic neuropeptide expression.

\section{Effects of hypothalamic ERK1/2 on glucose-regulated Pomc and Agrp/Npy expression in rats}

To further validate the role of ERK1/2 observed in vitro, food-deprived rats were given i.c.v. injections of glucose in the presence or absence of PD98059 and hypothalamic ARC tissues were collected $1 \mathrm{~h}$ later for protein and gene analyses. Consistent with results described in previous reports (Cha et al. 2008, Minokoshi et al. 2004), glucose injection suppressed the hypothalamic pAMPK (Fig. 6A), upregulated the Pomc and downregulated Agrp/Npy expression (Fig. 6B). It also markedly raised hypothalamic pERK1/2 levels (Fig. 6A) in agreement with the results of the in vitro experiments (Fig. 3). Administration of PD98059 dose-dependently depressed glucoseinduced ERK1/2 phosphorylation with no effect on

Published by Bioscientifica Ltd 
A
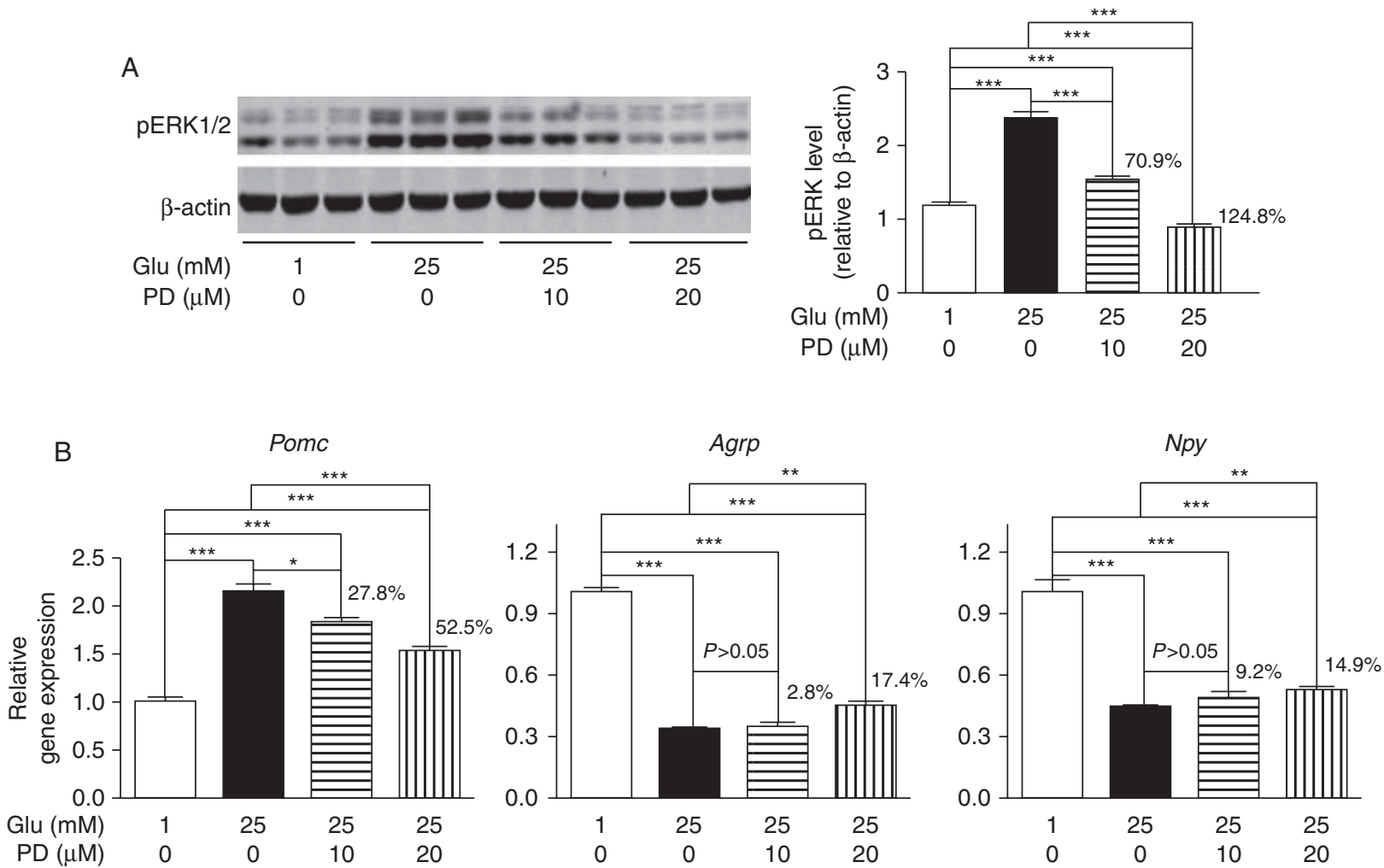

Figure 4

Effects of PD98059 on glucose-regulated Pomc and Agrp/Npy gene expression. DIV10 neurons were incubated in Neurobasal medium containing $1 \mathrm{mM}$ glucose overnight, followed by pretreatment with either DMSO or $10 \mu \mathrm{M}$ or $20 \mu \mathrm{M}$ PD98059 (PD) in Neurobasal medium containing $1 \mathrm{mM}$ glucose for $30 \mathrm{~min}$ before treatment with Neurobasal medium

pAMPK levels (Fig. 6A). PD98059 at $2.5 \mu \mathrm{g}$, at which $57 \%$ inhibition of pERK1/2 was achieved (Fig. 6A), blocked glucose-induced Pomc expression by 33\% and had no effect on Agrp/Npy expression (P>0.05, Fig. 6B). PD98059 at $5 \mu \mathrm{g}$, which gave a $123 \%$ inhibition of pERK1/2 levels, attenuated the Pomc expression by $57 \%$, whereas the glucose-suppressed Agrp/Npy expression was only modestly (17-19\%) reversed (Fig. 6B) despite an excess inhibition of the ERK1/2 activity. The i.c.v. dose of $5 \mu \mathrm{g}$ PD98059 has been used in food intake studies, and did not exhibit apparent toxicities in rats (Rahmouni et al. 2009). Thus the in vivo results confirm that ERK $1 / 2$ is an indispensible mediator in glucose-regulated Pomc but not Agrp/Npy expression.

\section{Discussion}

Hypothalamic neuroprogenitor cells have been used to study the molecular mechanisms of neuroendocrine hormones (Bergonzelli et al. 2001), leptin sensitivity in containing $25 \mathrm{mM}$ glucose in the presence or absence of PD98059 for $1 \mathrm{~h}$. The PERK $1 / 2$ level was analyzed by western blottings (A) and gene expression was analyzed by qPCR (B). Experiments were repeated at least twice and the data were consistent between experiments. ${ }^{*} P<0.05$, $* * P<0.01$, and $* * * P<0.001$.

developing hypothalamic neurons (Carlo et al. 2007), and hormonal effects on hypothalamic neural differentiation (Desai et al. 2011). The progenitor cells also express genes and proteins of feeding-related neuropeptides (Sousa-Ferreira et al. 2011). In this study, we established primary hypothalamic neurons from progenitor cells to study glucose sensing. Using hypothalamic cell models to study hypothalamic nutrient sensing could be particularly fitting because changes in extracellular nutrient levels directly trigger the cellular events (e.g., gene expression, electrical activity) without the mediation of neurotransmission. Nevertheless, it should be stressed that although DIV10 neurons are post-mitotic (i.e., expressing NeuN) and express leptin-regulated POMC and AgRP/NPY genes, which may make them more applicable than immortalized cell lines as a model system, they are not adult hypothalamic neurons (e.g., at minimum, expression levels of the few genes examined differ between cultured neurons and adult hypothalami). Besides, whether these neurons express other feeding-related neuropetides

Published by Bioscientifica Ltd 
A

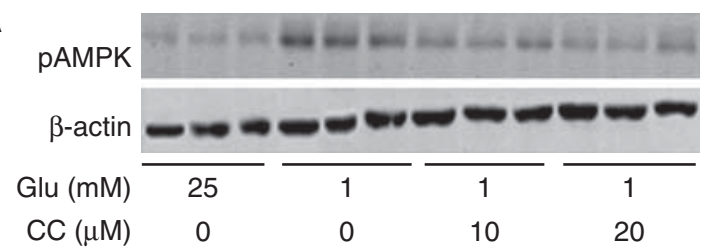

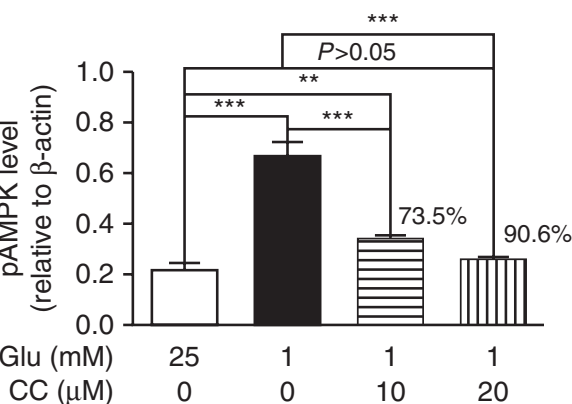

$\mathrm{CC}(\mu \mathrm{M})$

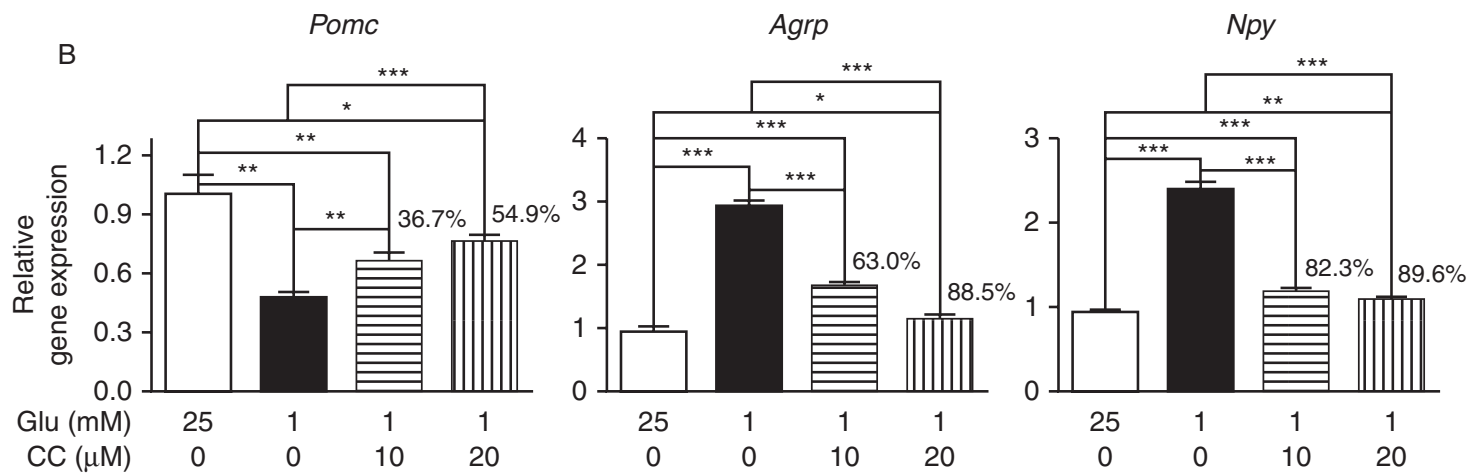

Figure 5

Effects of CC on glucose-regulated Pomc and Agrp/Npy gene expression. DIV10 neurons were pretreated with either DMSO or 10 or $20 \mu \mathrm{M} \mathrm{CC}$ in Neurobasal medium containing $25 \mathrm{mM}$ glucose for $30 \mathrm{~min}$ before treatment with Neurobasal medium containing $1 \mathrm{mM}$ glucose in the

(e.g., $\mathrm{MCH}$, orexin, and kisspeptin) or whether they represent a mixed population of hypothalamic neurons, as is the case in vivo, is not known. Nonetheless, because the results of in vivo experiments validate the findings obtained with the cultured neurons, the cell system may have utility for exploring the molecular details of hypothalamic nutrient sensing.

ARC is in close contact with the median eminence, a region characterized by a leaky blood-brain barrier (Banks 2006). Therefore, ARC neurons may be exposed to glucose concentrations close to those of the peripheral blood. In this study, cultured hypothalamic neurons detect a broad range of glucose levels $(1-25 \mathrm{mM})$ from clinical hypoglycemia to hyperglycemia and in turn produce a dynamic change in neuropeptide expression. The finding appears to differ from those obtained in electrophysiological studies, where the responsiveness of hypothalamic neurons to glucose concentrations is narrower and less dynamic. For instance, Pomc-expressing neurons hyperpolarize at glucose concentrations lowered from 10 to 5 or 5 to 3 , or 2 to $0.1 \mathrm{mM}$ (Ibrahim et al. 2003, Claret et al. 2007, Parton et al. 2007) and presence or absence of $\mathrm{CC}$ for $1 \mathrm{~h}$. Thereafter, the levels of pAMPK were analyzed by western blots (A) and gene expression analyzed by qPCR (B). Experiments were repeated at least twice and the results were consistent between experiments. ${ }^{*} P<0.05, * * P<0.01$, and $* * * P<0.001$.

NPY-expressing neurons depolarize at glucose levels dropping from 2.5 to $0.1 \mathrm{mM}$ or 15 to $1 \mathrm{mM}$ (Fioramonti et al. 2007, Mountjoy et al. 2007). Whether the differential glucose sensitivity between the two glucose-sensing readouts (gene expression and electrical activity) reflects differences in methodologies used or mechanisms requires future studies. It is conceivable though that the dynamic changes in appetitive gene expression in response to glucose signals would correlate well with the continuity in changes to physiological endpoints such as food intake and body weight.

Altered intracellular ATP levels are thought to be the main trigger to initiate signaling cascades in hypothalamic nutrient sensing (Blouet \& Schwartz 2010, Jordan et al. 2010). AMPK is activated by increased cellular AMP or decreased ATP levels (Kahn et al. 2005, Hardie et al. 2006). In $\beta$-cells, the glucose-induced ERK1/2 activation is also thought to be ATP-dependent, where glucose is taken up and metabolized to generate ATP, leading to a series of events including closure of the ATP-dependent potassium channel, activation of the voltage-dependent calcium channel, and the final release of calcium from intracellular

Published by Bioscientifica Ltd. 
A
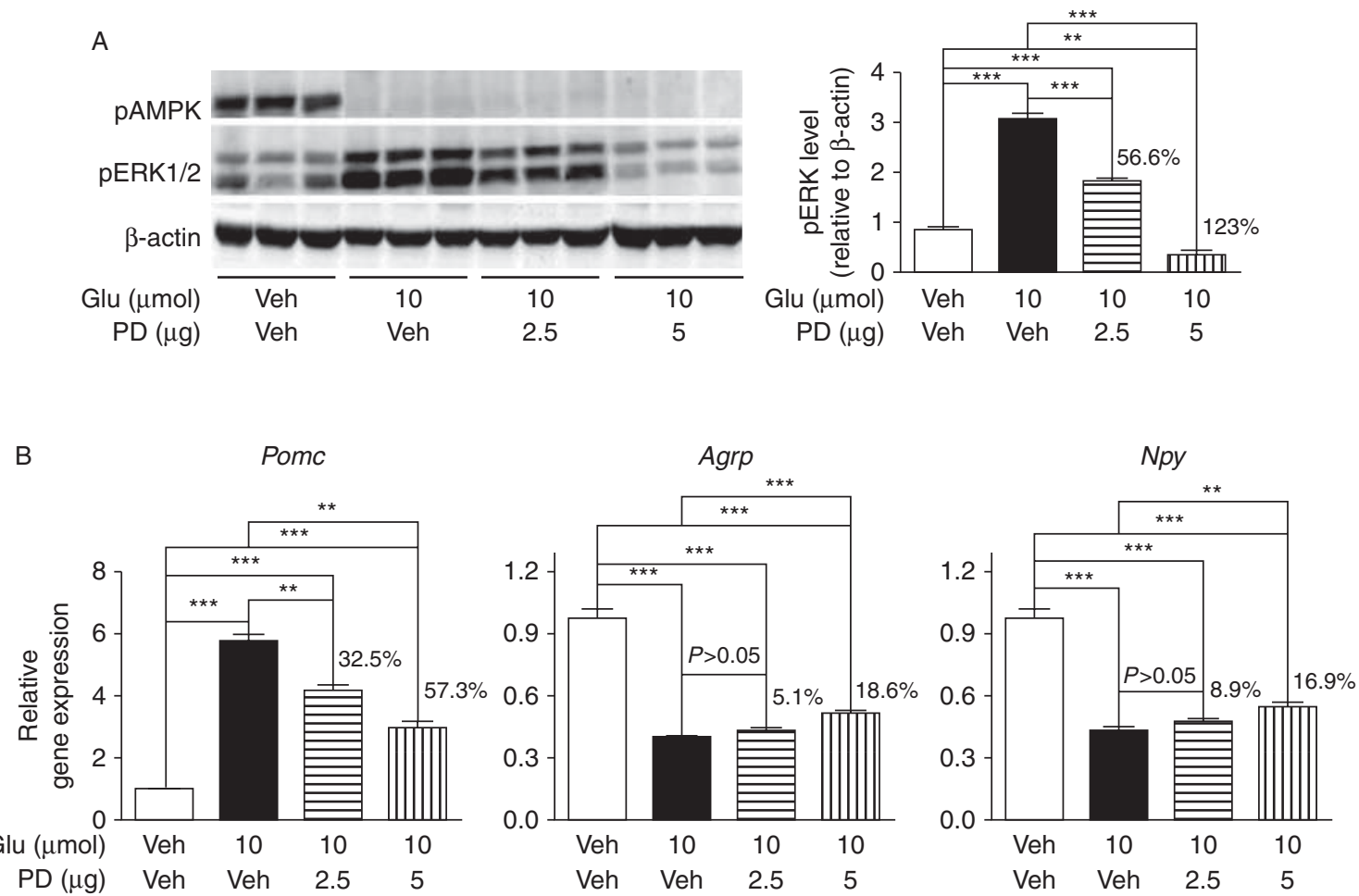

\section{Figure 6}

Effects of i.c.v. injection of PD98059 on glucose-induced ERK1/2 phosphorylation and feeding-related neuropeptide expression. Food-deprived rats were given i.c.v. injection of PD98059 $(2.5 \mu \mathrm{g}$ or $5 \mu \mathrm{g})$ or vehicle $30 \mathrm{~min}$

stores; a rise in intracellular calcium activates ERK1/2 (Lawrence et al. 2008). It is likely that nutrient metabolism also drives glucose-induced ERK1/2 activation in hypothalamic neurons. The common metabolism-dependent mechanism would explain the finding that glucose regulates AMPK and ERK1/2 activities in opposing directions. However, besides ATP as the shared trigger to either activate or deactivate the two signaling molecules, the two signaling pathways do not seem to be in tandem but parallel, as an ERK1/2-specific inhibitor had no effect on AMPK phosphorylation (Fig. 6A) or vice versus (results not shown).

We used pharmacological tools to modulate ERK1/2 or AMPK activities and showed that both ERK1/2 and AMPK signaling pathways are required for glucoseregulated $P O M C$ expression. Because the inhibitors used do not show apparent toxic effects under the similar conditions used in other studies (Alessi et al. 1995, Mountjoy et al. 2007, Rahmouni et al. 2009) and the inhibitors behaved as expected in this study (i.e., either increasing or decreasing gene expression), it is unlikely that the results are artifacts. Our findings thus may provide before the i.c.v. administration of glucose $(10 \mu \mathrm{mol})$. The rat hypothalami were removed 1-h post glucose treatment for western blotting $(A)$ and qPCR analyses (B). ${ }^{*} P<0.05, * * P<0.01$, and $* * * P<0.001$.

an explanation for earlier perplexing observations. For instance, Pomc $\alpha 2 \mathrm{KO}$ transgenic mice display a paradoxical phenotype of increased feeding and body weight (Claret et al. 2007). Here, we show that there are dual ERK1/2 and AMPK signaling pathways exerting opposite effects on Pomc expression. It is possible that a compensatory suppression of the ERK1/2 pathway leading to an overinhibition of Pomc expression in Pomc 2 KO mice might have led to the discordant phenotypes, especially it has been suggested that hypothalamic circuits are biased towards weight gain (Schwartz et al. 2003, Morton et al. 2006). It is also attractive to speculate that ERK1/2 might participate in the glucose-regulated membrane potential change of POMC neurons. A clear role of AMPK in the electrical activity of basomedial hypothalamic glucoseexcited (including Pomc-expressing) neurons (Mountjoy et al. 2007) could have been masked by the presence of ERK1/2 activities.

In this study, we showed the acute effect $(1 \mathrm{~h})$ of ERK1/2 on glucose-regulated Pomc expression. The phosphorylation of ERK1/2 and Pomc expression in DIV10 neurons also remained high after 6 -h glucose treatment

Published by Bioscientifica Ltd. 
(data not shown). However, whether ERK1/2 exerts a longer-term effect on Pomc expression remains to be examined. It is also not clear how ERK1/2 differentially mediates glucose-regulated orexigenic and anorexigenic neuropeptide expression. Because both Pomc and Agrp/Npy neurons express ERK1/2 (Morikawa et al. 2004, Rahmouni et al. 2009), it is possible that the difference may reside in their promoter regions. Two glucose-responsive elements (A and $\mathrm{E}$ boxes) in the insulin promoter drive the ERK1/ 2 -mediated insulin expression in $\beta$-cells (Lawrence et al. 2008). Whether such DNA elements are present in the Pomc promoter (and cryptic/absent in the Agrp/Npy promoters) would be worth exploring.

In conclusion, using primary hypothalamic neurons and pharmacological tools we investigated the molecular mediators relaying glucose signals to the expression of feeding-related neuropeptides. Our data establish for the first time, to our knowledge, that AMPK mediates glucoseregulated $P O M C$ expression in manners as proposed. In addition, the results indicate ERK1/2 to be a novel co-mediator governing POMC expression. As the in vitro data is supported by the in vivo results, cultured hypothalamic neurons may have utility for studying hypothalamic nutrient sensing.

\section{Declaration of interest}

The authors declare that there is no conflict of interest that could be perceived as prejudicing the impartiality of the research reported.

\section{Funding}

This work was supported by the Overseas Recruitment Fund for Universities in Guangdong, China.

\section{Acknowledgements}

We thank Professor Haiyun Xu at Shantou University Medical College for providing the stereotaxic apparatus and technical guidance to stereotaxic injection.

\section{References}

Alessi DR, Cuenda A, Cohen P, Dudley DT \& Saltiel AR 1995 PD 098059 is a specific inhibitor of the activation of mitogen-activated protein kinase kinase in vitro and in vivo. Journal of Biological Chemistry 270 27489-27494. (doi:10.1074/jbc.270.46.27489)

Banks WA 2006 Blood-brain barrier and energy balance. Obesity 14 (Suppl 5) 234s-237s. (doi:10.1038/oby.2006.315)

Barsh GS \& Schwartz MW 2002 Genetic approaches to studying energy balance: perception and integration. Nature Reviews. Genetics $\mathbf{3}$ 589-600. (doi:10.1038/nrn902)

Bergonzelli GE, Pralong FP, Glauser M, Cavadas C, Grouzmann E \& Gaillard RC 2001 Interplay between galanin and leptin in the hypothalamic control of feeding via corticotropin-releasing hormone and neuropeptide Y. Diabetes 50 2666-2672. (doi:10.2337/diabetes. 50.12.2666)

Blouet C \& Schwartz GJ 2010 Hypothalamic nutrient sensing in the control of energy homeostasis. Behavioural Brain Research 209 1-12. (doi:10.1016/j.bbr.2009.12.024)

Cai F, Gyulkhandanyan AV, Wheeler MB \& Belsham DD 2007 Glucose regulates AMP-activated protein kinase activity and gene expression in clonal, hypothalamic neurons expressing proopiomelanocortin: additive effects of leptin or insulin. Journal of Endocrinology 192 605-614. (doi:10.1677/JOE-06-0080)

Carlo AS, Pyrski M, Loudes C, Faivre-Baumann A, Epelbaum J, Williams LM \& Meyerhof W 2007 Leptin sensitivity in the developing rat hypothalamus. Endocrinology 148 6073-6082. (doi:10.1210/ en.2007-0822)

Cha SH, Wolfgang M, Tokutake Y, Chohnan S \& Lane MD 2008 Differential effects of central fructose and glucose on hypothalamic malonyl-CoA and food intake. PNAS 105 16871-16875. (doi:10.1073/pnas. 0809255105)

Chang GQ, Karatayev O, Davydova Z \& Leibowitz SF 2004 Circulating triglycerides impact on orexigenic peptides and neuronal activity in hypothalamus. Endocrinology 145 3904-3912. (doi:10.1210/en. 2003-1582)

Chang GQ, Karatayev O, Davydova Z, Wortley K \& Leibowitz SF 2005 Glucose injection reduces neuropeptide $\mathrm{Y}$ and agouti-related protein expression in the arcuate nucleus: a possible physiological role in eating behavior. Brain Research. Molecular Brain Research 135 69-80. (doi:10.1016/j.molbrainres.2004.12.017)

Cheng H, Isoda F, Belsham DD \& Mobbs CV 2008 Inhibition of agoutirelated peptide expression by glucose in a clonal hypothalamic neuronal cell line is mediated by glycolysis, not oxidative phosphorylation. Endocrinology 149 703-710. (doi:10.1210/ en.2007-0772)

Claret M, Smith MA, Batterham RL, Selman C, Choudhury AI, Fryer LG, Clements M, Al-Qassab H, Heffron H, Xu AW et al. 2007 AMPK is essential for energy homeostasis regulation and glucose sensing by POMC and AgRP neurons. Journal of Clinical Investigation 117 2325-2336. (doi:10.1172/JCI31516)

Cone RD 2005 Anatomy and regulation of the central melanocortin system. Nature Neuroscience 8 571-578. (doi:10.1038/nn1455)

Desai M, Li T \& Ross MG 2011 Fetal hypothalamic neuroprogenitor cell culture: preferential differentiation paths induced by leptin and insulin. Endocrinology 152 3192-3201. (doi:10.1210/en.2010-1217)

Fioramonti X, Contie S, Song Z, Routh VH, Lorsignol A \& Penicaud L 2007 Characterization of glucosensing neuron subpopulations in the arcuate nucleus: integration in neuropeptide $\mathrm{Y}$ and pro-opio melanocortin networks? Diabetes 56 1219-1227. (doi:10.2337/db06-0567)

Hardie DG, Hawley SA \& Scott JW 2006 AMP-activated protein kinase development of the energy sensor concept. Journal of Physiology $\mathbf{5 7 4}$ 7-15. (doi:10.1113/jphysiol.2006.108944)

Ibrahim N, Bosch MA, Smart JL, Qiu J, Rubinstein M, Ronnekleiv OK, Low MJ \& Kelly MJ 2003 Hypothalamic proopiomelanocortin neurons are glucose responsive and express $\mathrm{K}_{\mathrm{ATP}}$ channels. Endocrinology 144 1331-1340. (doi:10.1210/en.2002-221033)

Jordan SD, Konner AC \& Bruning JC 2010 Sensing the fuels: glucose and lipid signaling in the CNS controlling energy homeostasis. Cellular and Molecular Life Sciences 67 3255-3273. (doi:10.1007/s00018010-0414-7)

Kahn BB, Alquier T, Carling D \& Hardie DG 2005 AMP-activated protein kinase: ancient energy gauge provides clues to modern understanding of metabolism. Cell Metabolism 1 15-25. (doi:10.1016/j.cmet.2004. 12.003)

Khoo S, Griffen SC, Xia Y, Baer RJ, German MS \& Cobb MH 2003 Regulation of insulin gene transcription by ERK1 and ERK2 in pancreatic $\beta$ cells. Journal of Biological Chemistry 278 32969-32977. (doi:10.1074/jbc. M301198200) 
Kurata K, Fujimoto K, Sakata T, Etou H \& Fukagawa K 1986 D-glucose suppression of eating after intra-third ventricle infusion in rat. Physiology \& Behavior 37 615-620. (doi:10.1016/0031-9384(86)90295-7)

Lawrence M, Shao C, Duan L, McGlynn K \& Cobb MH 2008 The protein kinases ERK1/2 and their roles in pancreatic beta cells. Acta Physiologica 192 11-17. (doi:10.1111/j.1748-1716.2007.01785.x)

Lee K, Li B, Xi X, Suh Y \& Martin RJ 2005 Role of neuronal energy status in the regulation of adenosine 5'-monophosphate-activated protein kinase, orexigenic neuropeptides expression, and feeding behavior. Endocrinology 146 3-10. (doi:10.1210/en.2004-0968)

Martins PJ, Haas M \& Obici S 2010 Central nervous system delivery of the antipsychotic olanzapine induces hepatic insulin resistance. Diabetes 59 2418-2425. (doi:10.2337/db10-0449)

Minokoshi Y, Alquier T, Furukawa N, Kim YB, Lee A, Xue B, Mu J, Foufelle F, Ferre P, Birnbaum MJ et al. 2004 AMP-kinase regulates food intake by responding to hormonal and nutrient signals in the hypothalamus. Nature 428 569-574. (doi:10.1038/nature02440)

Morikawa Y, Ueyama E \& Senba E 2004 Fasting-induced activation of mitogen-activated protein kinases (ERK/p38) in the mouse hypothalamus. Journal of Neuroendocrinology 16 105-112. (doi:10.1111/j. 0953-8194.2004.01135.x)

Morton GJ, Cummings DE, Baskin DG, Barsh GS \& Schwartz MW 2006 Central nervous system control of food intake and body weight. Nature 443 289-295. (doi:10.1038/nature05026)

Mountjoy PD \& Rutter GA 2007 Glucose sensing by hypothalamic neurones and pancreatic islet cells: AMPle evidence for common mechanisms? Experimental Physiology 92 311-319. (doi:10.1113/ expphysiol.2006.036004)

Mountjoy PD, Bailey SJ \& Rutter GA 2007 Inhibition by glucose or leptin of hypothalamic neurons expressing neuropeptide Y requires changes in AMP-activated protein kinase activity. Diabetologia 50 168-177. (doi:10.1007/s00125-006-0473-3)

Parton LE, Ye CP, Coppari R, Enriori PJ, Choi B, Zhang CY, Xu C, Vianna CR, Balthasar N, Lee CE et al. 2007 Glucose sensing by POMC neurons regulates glucose homeostasis and is impaired in obesity. Nature $\mathbf{4 4 9}$ 228-232. (doi:10.1038/nature06098)

Rahmouni K, Sigmund CD, Haynes WG \& Mark AL 2009 Hypothalamic ERK mediates the anorectic and thermogenic sympathetic effects of leptin. Diabetes 58 536-542. (doi:10.2337/db08-0822)

Schuit FC, Huypens P, Heimberg H \& Pipeleers DG 2001 Glucose sensing in pancreatic $\beta$-cells: a model for the study of other glucose-regulated cells in gut, pancreas, and hypothalamus. Diabetes 50 1-11. (doi:10.2337/ diabetes.50.1.1)

Schwartz MW, Woods SC, Seeley RJ, Barsh GS, Baskin DG \& Leibel RL 2003 Is the energy homeostasis system inherently biased toward weight gain? Diabetes 52 232-238. (doi:10.2337/diabetes.52.2.232)

Sousa-Ferreira L, Alvaro AR, Aveleira C, Santana M, Brandao I, Kugler S, de Almeida LP \& Cavadas C 2011 Proliferative hypothalamic neurospheres express NPY, AGRP, POMC, CART and Orexin-A and differentiate to functional neurons. PLOS ONE 6 e19745. (doi:10.1371/journal.pone. 0019745)

Toda C, Shiuchi T, Kageyama H, Okamoto S, Coutinho EA, Sato T, Okamatsu-Ogura Y, Yokota S, Takagi K, Tang L et al. 2013 Extracellular signal-regulated kinase in the ventromedial hypothalamus mediates leptin-induced glucose uptake in red-type skeletal muscle. Diabetes 62 2295-2307. (doi:10.2337/db12-1629)

Ueyama E, Morikawa Y, Yasuda T \& Senba E 2004 Attenuation of fasting-induced phosphorylation of mitogen-activated protein kinases (ERK/p38) in the mouse hypothalamus in response to refeeding. Neuroscience Letters 371 40-44. (doi:10.1016/j.neulet.2004. 08.035)

Varela L \& Horvath TL 2012 Leptin and insulin pathways in POMC and AgRP neurons that modulate energy balance and glucose homeostasis. EMBO Reports 13 1079-1086. (doi:10.1038/embor.2012.174)

Wolfgang MJ, Cha SH, Sidhaye A, ChohnanS, Cline G, Shulman GI \& Lane MD 2007 Regulation of hypothalamic malonyl-CoA by central glucose and leptin. PNAS 104 19285-19290. (doi:10.1073/pnas.0709778104)

Received in final form 23 January 2015

Accepted 26 January 2015

Accepted Preprint published online 26 January 2015
(C) 2015 Society for Endocrinology Printed in Great Britain 